\title{
Correction to: Thermal Expansion, Elastic and Magnetic Properties of FeCoNiCu-Based High-Entropy Alloys Using First-Principle Theory
}

\author{
SHUO HUANG, ${ }^{1,6}$ ÁDÁM VIDA,,${ }^{2,3}$ ANITA HECZEL, ${ }^{3}$ ERIK HOLMSTRÖM, ${ }^{4}$ \\ and LEVENTE VITOS ${ }^{1,2,5}$ \\ 1.-Applied Materials Physics, Department of Materials Science and Engineering, Royal Institute \\ of Technology, 10044 Stockholm, Sweden. 2.-Institute for Solid State Physics and Optics, Wigner \\ Research Centre for Physics, P.O. Box 49, Budapest 1525, Hungary. 3.-Department of Materials \\ Physics, Eötvös University, Pázmány Péter Sétány 1/A, Budapest 1117, Hungary. 4.-Sandvik \\ Coromant R\&D, 12680 Stockholm, Sweden. 5.-Department of Physics and Astronomy, Division \\ of Materials Theory, Uppsala University, Box 516, 75120 Uppsala, Sweden. 6.-e-mail: \\ shuoh@kth.se
}

\section{Correction to:}

JOM, Vol. 69, No. 11, 2017

https://doi.org/10.1007/s11837-017-2565-6

This article was originally published Online First without open access. After publication in volume 69 , issue 11, page 2107-2112 the author decided to opt for Open Choice and to make the article an open access publication. Therefore, the copyright of the article has been changed to (c) The Author(s) 2017 and the article is forthwith distributed under the terms of the Creative Commons Attribution 4.0 International License (http://creativecommons.org/ licenses/by/4.0/), which permits use, duplication, adaptation, distribution and reproduction in any medium or format, as long as you give appropriate credit to the original author(s) and the source, provide a link to the Creative Commons license, and indicate if changes were made. The original article was corrected. 